\title{
Development of Ankle Foot Orthosis (AFO) Using Pneumatic Artificial Muscle for Disabled Children
}

\author{
N.Z. Ishak ${ }^{1}$, S. Mohamaddan ${ }^{1, a}$, A.M.N.A Kamaruddin ${ }^{1}$, H. Khamis ${ }^{1}$, S. Yamamoto ${ }^{2}$ and S.Z.M. Dawal ${ }^{3}$ \\ ${ }^{1}$ Department of Mechanical and Manufacturing Engineering, Faculty of Engineering, Universiti Malaysia Sarawak (UNIMAS), 94300 \\ Kota Samarahan, Sarawak, Malaysia \\ ${ }^{2}$ Department of Bioscience and Engineering, College of System Engineering and Science, Shibaura Institute of Technology, 3308570 \\ Saitama, Japan \\ ${ }^{3}$ Deparment of Mechanical Engineering, Faculty of Engineering, University of Malaya, 50603 Kuala Lumpur, Malaysia
}

\begin{abstract}
Ankle foot orthosis (AFO) are commonly used to correct the instabilities and joint weakness of lower limb. In this research, AFO was developed by using pneumatic artificial muscle (PAM) to prevent plantarflexion to occur and also to correct the foot from the inversion syndrome. The research started with designing the AFO by using SolidWorks software based on anthropometry measurement data $(\mathrm{n}=5$, age $=12$ years old). The mechanical simulation was conducted by using Autodesk Inventor software to obtain a safety factor before the fabrication process was conducted. The AFO was fabricated using 3D printer and the thermoplastic elastomer (TPE) rubber was selected as the material. PAM was tested by using test bed machine to generate the force and contraction by muscle. The result shows that the PAM was suitable for low speed as the displacement was greater. The AFO could be valuable for the gait rehabilitation.
\end{abstract}

\section{Introduction}

Disabled children with foot drop syndrome have difficulties to walk properly due to plantarflexion during swing phase as their long nerves are damage. This research concern about the plantarflexion because the muscles are important to support the center of mass [1]. The children may have a tendency to walk on the toes as the affected foot striking the ground first instead of the usual heel strike [2]. Furthermore, they will be dragging their toes in semicircle and the foot will slap down onto the floor.

The foot syndrome behavior will give harmful effect to their leg. In this research, the gait cycle of foot drop is concerned. Gait cycle can be defined as a walking manner of a person and can be divided into two parts; stance and swing phase. In normal gait cycle, stance phase covers $60 \%$ of the walking movement that begins with right foot of the heel strike until the toe off. The other $40 \%$ is considered as swing phase, and the walking movement is between the toe off and the heel strike [3].

Dorsiflexion and plantarflexion are occurred during walking cycle. In normal gait, the dorsiflexion is needed in order to prevent foot drop during stance phase, while the plantarflexion takes place to push the toe or foot upward. However, the dorsiflexion and plantarflexion have a certain angle in ankle behavior during regular gait as shown in Figure 1. If the ankle angle is excessive than the normal angle, that is where the abnormal gait cycle is happened.

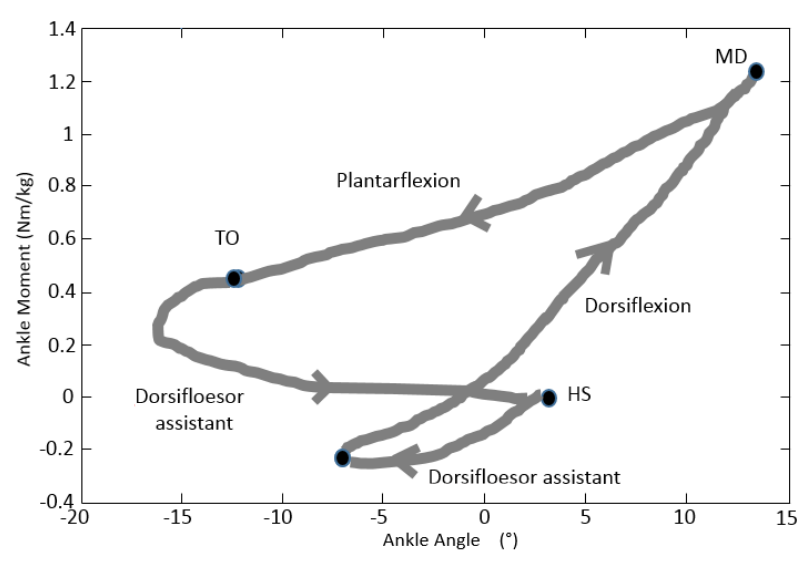

Figure 1. Stiffness characteristic of ankle of a normal gait. HS: Hill strike, FF: Flat foot, MD: Maximum dorsiflexion, and TO: Toe off [4]

Besides foot drop, inversion and eversion also taken into account in this research. Inversion and eversion can occurred when the ligaments are stretched out far from its ligament. An inversion is the movements where the sole of the foot is facing inwards while eversion is the movements where the sole of the foot is facing outwards. Inversion occurs more often compared to eversion. Due to this, this research put forward a new design of AFO device that could be used to prevent plantarflexion and also to prevent inversion from occurred.

\footnotetext{
a Corresponding author: mshahrol@unimas.my
} 
The more widely device that is used for foot drop treatment is ankle foot orthosis (AFO). An AFO is a device or brace that covered the foot, ankle and also part of leg [5]. This device is used to correct the instabilities and joint weaknesses of lower limb muscle [6]. Utilizing AFO will improve the mobility or can be used as rehabilitation devices to correct their motor patterns [7]. Besides, by using the device, user may experience improvement of the walking pattern performance by proper control motion of the device.

AFO can be divided into three groups which are passive, semi-active and fully active [8]. Passive AFO is normally used to fix or articulated the joints, while semi active AFO is used to modulate damping at the joints and lastly fully active AFO is used to produce torque for propulsive assistance and control the motion.

Passive AFO has the absence of the control or onboard electronics, but this type of AFO has a mechanical element. The mechanical element such as damper or spring can be used in order to control the ankle joint's motion during gait performance [8]. This type of AFO is commonly used to treat the patient with weakness muscle at ankle joint and it can be used as a daily wear assistive device.

The semi-active types of AFO utilized the computer control for varying compliance or the joint damping in real time [8]. Besides, the AFO can be used for daily devices as it is not as heavy and complex as fully active AFO. Semi-active AFO depends on the feedback sensor for swing and stance phase, has untethered power sources and additional functional assistance.

Fully active AFO is usually used in laboratory research or center based rehabilitation as the design is more complex compared to passive and semi active AFO. The active AFO is used as training device where it helps the patient to improve their gait performance. Moreover, it can also be used as an instrument to measure the force and the motion of ankle joint and to the perturb gait in locomotion studies [9].

In this research, AFO device is actuated by pneumatic artificial muscle (PAM) that could prevent plantarflexion and inversion to occur. PAM is one type of actuator that contractile and linear motion, operated usually by air pressure which fills a pneumatic bladder [10]. As the PAM is pressurized, the PAM will inflate and shortens and when the gas is vented out, the membrane will squeeze. McKibben muscle type is used for the AFO design as it is lightweight, capable of high forces and can mimics or has similarity to the human skeletal muscles' behavior.

The anthropometric measurement is taken in order to design AFO. The anthropometric data is used and associated with product design and fabrication. Hence, the objectives in this research is to design and fabricate the AFO for disabled children and to develop a pneumatic artificial muscle in order to actuate the AFO.

\section{Methodology}

5 normal children, 3 boys and 2 girls, aged 12 years old, had participated in this study.

\subsection{Anthropometry Measurement}

18 dimensions of lower limb were taken in total of 5 subjects in the age of 12 years old for both boys and girls as shown in Table 1. These dimensions are useful in designing the AFO. Dimensions were measured by using standard professional anthropometric tools (Rosscraft, Canada).

A sliding caliper was used to measure small breadths and depths body segment, a weighing scale used to measure human weight, a stadiometer used to measure human height, a plastic measuring tape was used to measure body circumferences and an adjustable chair for sitting straight lines, curves, circumferences, and thickness. The recorded anthropometric data is then used to associate with the product design and fabrication.

Table 1 Anthropometric measurement data [11]

\begin{tabular}{lccc}
\hline \multicolumn{1}{c}{ Dimension } & $\mathbf{n = 5}$ & & \\
\cline { 2 - 4 } & Mean & SD & $\mathbf{C V ( \% )}$ \\
\hline 1. Tibia Length & 32.59 & 3.60 & 4.38 \\
2. Calf girth & 30.20 & 5.09 & 4.13 \\
3. Knee girth & 31.26 & 3.98 & 2.87 \\
4. Ankle girth & 18.82 & 2.08 & 4.65 \\
5. Foot length & 21.20 & 1.58 & 1.91 \\
6. Ball of foot length & 15.63 & 1.49 & 5.08 \\
7. Outside ball of foot & 13.74 & 1.12 & 6.74 \\
length & & & \\
8. Foot breath & 8.74 & 0.82 & 3.17 \\
$\quad$ diagonal & & & \\
9. Foot breath & 8.70 & 0.86 & 2.71 \\
$\quad$ horizontal & & & \\
10. Heel breadth & 5.41 & 0.78 & 7.02 \\
11. Ball girth & 20.69 & 1.52 & 1.93 \\
12. Instep girth & 20.30 & 1.91 & 3.73 \\
13. Short heel girth & 24.66 & 9.98 & 3.65 \\
14. Sphyrion height & 8.43 & 0.39 & 4.42 \\
15. Sphyrion fibular & 7.40 & 0.72 & 10.81 \\
$\quad$ height & & & \\
16. Navicular height & 5.61 & 0.43 & 6.86 \\
17. Toe height & 1.52 & 0.10 & 5.34 \\
18. Instep height & 4.63 & 0.49 & 11.03 \\
\hline
\end{tabular}

\subsection{Mechanical Design}

A high number of fabrications are needed on typical AFO actuated by pneumatic artificial muscle (PAM). Normally, AFO is used to avoid plantarflexion to occur or assist dorsiflexion during swing phase. However, this research presents a new AFO device that could be used to prevent plantarflexion for foot drop, also providing eversion motion, which is useful for rehabilitation. In order to design the AFO, the anthropometric data was applied during design AFO on the right lower limb by using SolidWorks software.

All the design was transferred to the Autodesk Inventor Professional software in order to do a test simulation. This simulation is needed to simulate the design in a real condition before manufacturing. The design is then amended if the simulation shows a critical failure that needs to be addressed in order to improve the device safety and also built quality. 
In this research, 3D printer was used in order to fabricate the AFO. This process enables AFO to be custom built to each subject's right lower limb based on the anthropometric data collected earlier.

\subsection{Developing the PAM}

The PAM (McKibben muscle) is a simple and low cost approach to actuate the designed APO. In this design, desired for PAM length is $\mathrm{L}=250 \mathrm{~mm}$. The muscle consists of a rubber tube of $25 \mathrm{~mm}$ in diameter by using formula $(\mathrm{L}+30 \mathrm{~mm})$ and be surrounded by a FLEXO PET (mesh stripe) also of $25 \mathrm{~mm}$ in diameter with formula $(\mathrm{L}+40 \mathrm{~mm})$ as shown in Figure 2.

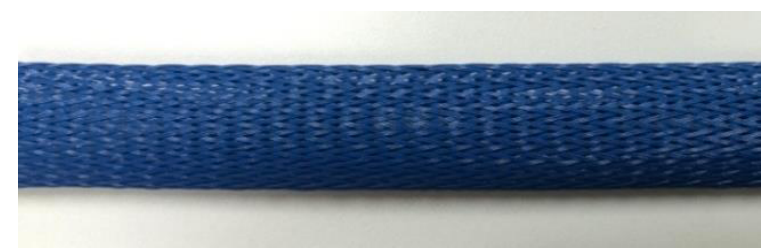

Figure 2. Mesh stripe (FLEXO PET 1inch, TECHFLEX)

Custom-designed shaft components were used in PAM assembly. To start the assembly, these shaft components are placed at the both ends of the rubber tube. The mesh stripe is pulled to cover the rubber tube. Then the copper ring $(21 \mathrm{~mm}$ outer diameter, $19 \mathrm{~mm}$ inner diameter) is placed at the both ends of the mesh stripe for finishing. Three jaws chuck used to tighten the copper ring to prevent air leaks during testing as shown in Figure 3. Finally, the copper ring is covered by tape to ensure no separation or pulls off occurs during bed testing.

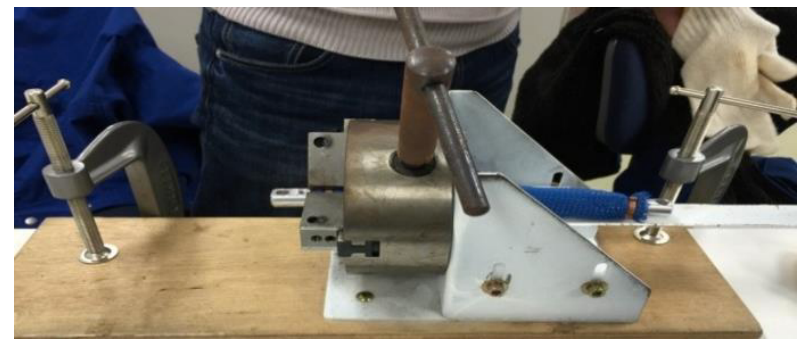

Figure 3. Three jaws chuck

The assembled PAM is then tested by on test bed as shown in Figure 4. This test bed is able to generate force by muscle and test the contraction by muscle. The test bed consists of a compressor (IWATA SLP-22) that supplies the air to PAM, a regulator (SMC PSE540AR06) to regulate or maintain air, laser displacement meter (OMRON ZX-SLD300L) to measure the displacement on PAM during contraction and inflation, load cell (KYOWA LUR-A-2KNSA1) for measuring tension or compression loads, light reflector (OMRON E39-RS1) to measure the reflection of laser for displacement occurred, force magnitudes produced during PAM extension were amplified by a strain amplifier (KYOWA DPM-611B) and lastly (NATIONAL INSTRUMENT DAQ NI cDAQ9178) to record the acquired data from the test.

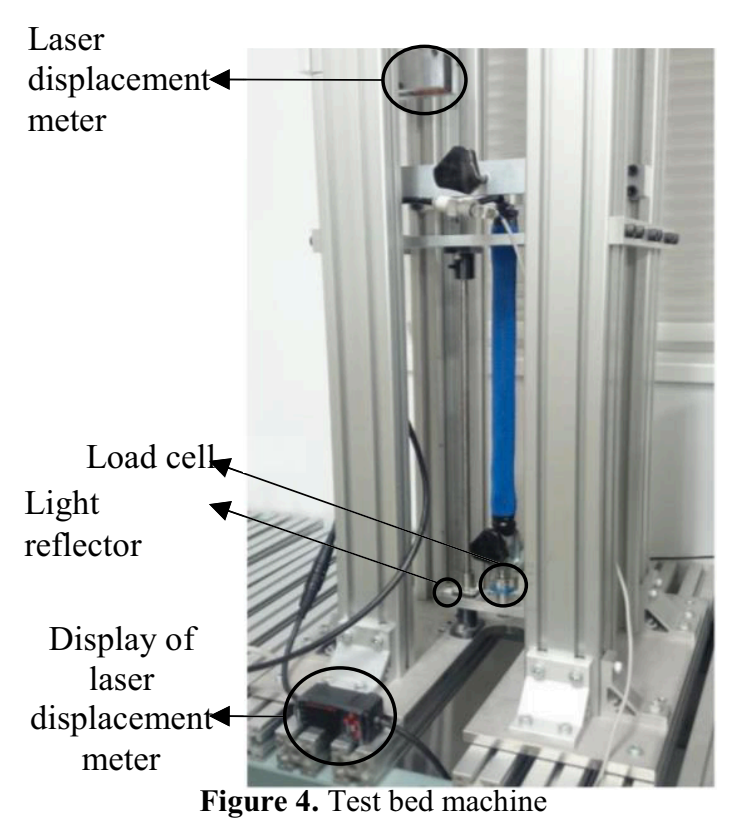

\subsection{Orthosis Control System}

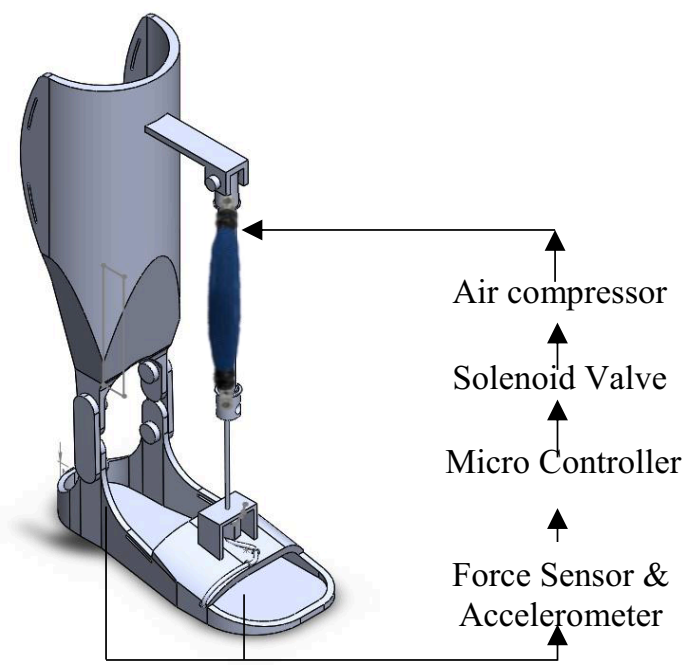

Figure 5. Control System Scheme

The control system scheme is shown in Figure 5, the pressure in PAM is controlled through the force resistive sensor (FRS). As the force is applied to the FRS sensor, the air from the opening space is being pushed and the conductive material make a contact with the active area parts. As this sensor changes its resistance with force, as the active area touches the conductive area activity increases, the lower its resistance thus low resistance to electric current. The sensor send signals to a micro controller (Arduino) to connect to the motor driver. These control signals are directed to solenoid valve that supply air to activate the PAM. Ankle angle, plantarflexion angle and artificial muscle force are recorded every minute during 5 full strides by using an encoder. 


\section{Results and Discussion}

\subsection{Design, Simulation and Fabrication of AFO}

Anthropometric data from Table 1 were applied during the designing AFO in SolidWorks software as shown in Figure 6 (a). However, as mentioned earlier, a rather high number of fabrications are needed for a typical AFO actuated by PAM. In the final design, instead of providing dorsiflexion for foot drop, the newly designed AFO could also provide eversion motion, which is useful for rehabilitation. In this research, from the anthropometric data of 18 dimensions in Table 1 , only 12 dimensions were needed in the new AFO design.

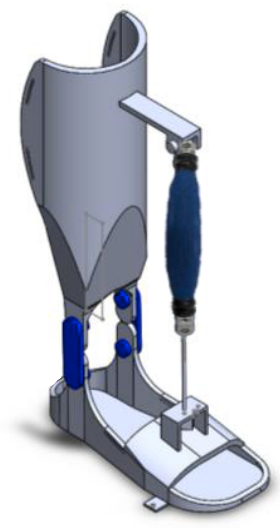

(a)

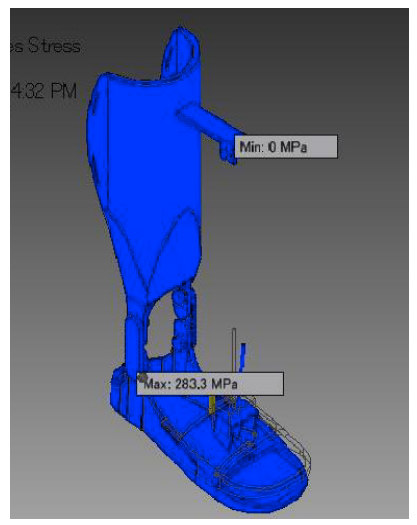

(b)
Figure 6(a). Ankle foot orthosis design by using SolidWorks software 6(b). Simulation by using Autodesk Inventor

The simulation analysis was performed with the pressure of $343.233 \mathrm{~N}(35 \mathrm{~kg})$ which is the average of children weight to be applied to the design. Three analyses which are von Mises stress, displacement and safety factor of this simulation are obtained. From the simulation result, the von Mises stress value is at 283.26MPa (maximum) as shown in Figure 6(b). The displacement for this design is $7.89 \mathrm{~mm}$ and the safety factor is at $15 \mathrm{ul}$ (maximum) and 1.36ul (minimum), which are fairly safe for user to wear with better quality of parts to be manufacture.
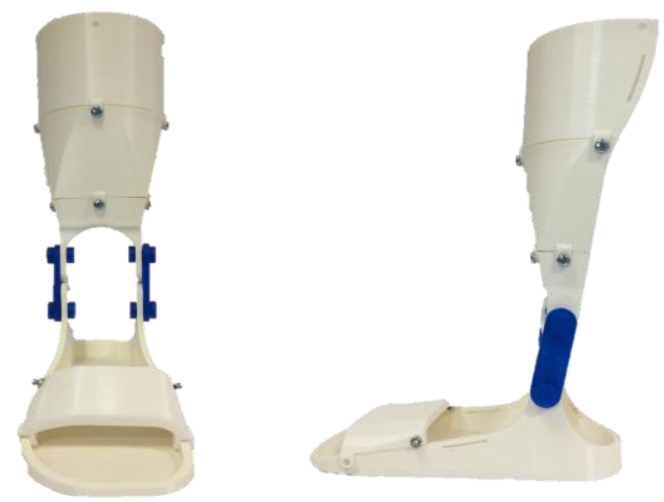

Figure 7. Fabrication of AFO by using 3D printer

After the simulation, the AFO is fabricated in order to custom fit each subject's their right lower limb. The construction of AFO is made of thermoplastic elastomer
(TPE) rubber by using 3D printer for both shank and foot section. A hinge between the shank and foot section is created by using polylactic acid (PLA) material to provide a stable AFO device, thus allowing a free ankle movement during stance phase. The fabricated AFO is shown in Figure 7.

\subsection{PAM Results}

The PAM has been examined by using the test bed experiment with the PAM length $250 \mathrm{~mm}$. This test bed is used to generate force by muscle and test the contraction by muscle. By using the average 12 years-old children weight ( $35 \mathrm{~kg})$, the foot mass is obtained by applying the formula of $0.0145 \mathrm{M}$ which is $0.5075 \mathrm{~kg}$ [8] as a benchmark.

Figure 8 shows of $0 \mathrm{~kg}$ load, (a) for $0.1 \mathrm{~Hz}$ obtain the displacement is $35 \mathrm{~mm}$ and (b) $15 \mathrm{~mm}$ for $1.0 \mathrm{~Hz}$ during the test bed inflation and contraction of pneumatic artificial muscle.

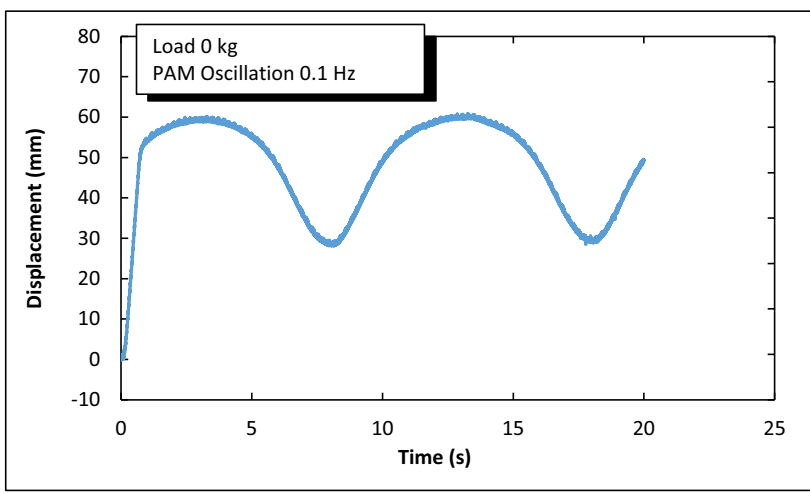

(a)

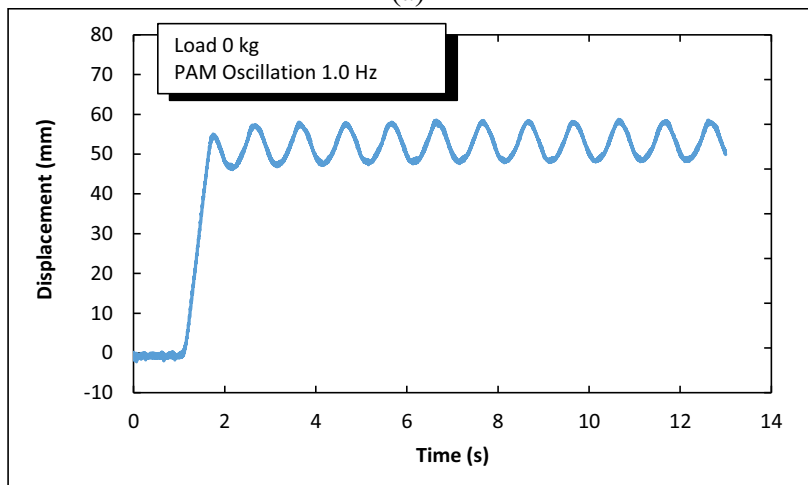

(b)

Figure 8. PAM test bed displacement experiment with $0 \mathrm{~kg}$ and a) $0.1 \mathrm{~Hz}$ and b) $1.0 \mathrm{~Hz}$.

The $0.1 \mathrm{~Hz}$ obtained the linear system in Figure 9(a) for PAM which is good indicator for a PAM system while, $1.0 \mathrm{~Hz}$ give nonlinear system Figure 9(b) due to the high speed. However, the nonlinear system for high speed could be explored for further research as the result produce at this stage is complicated. 


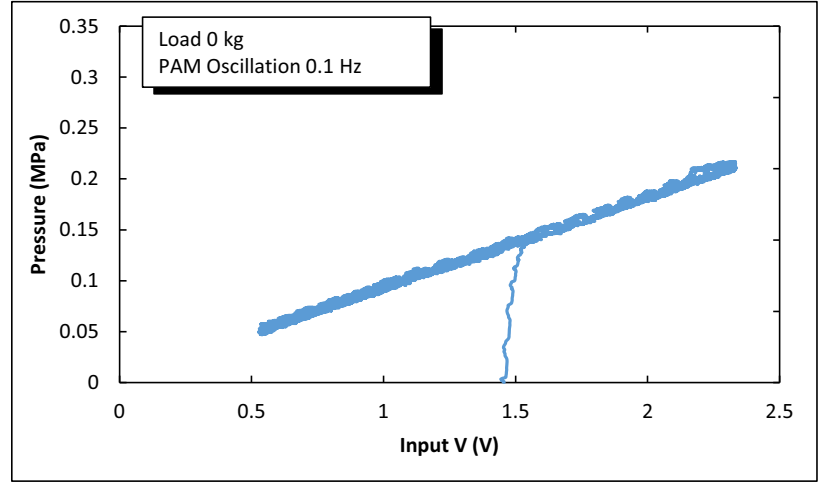

(a)

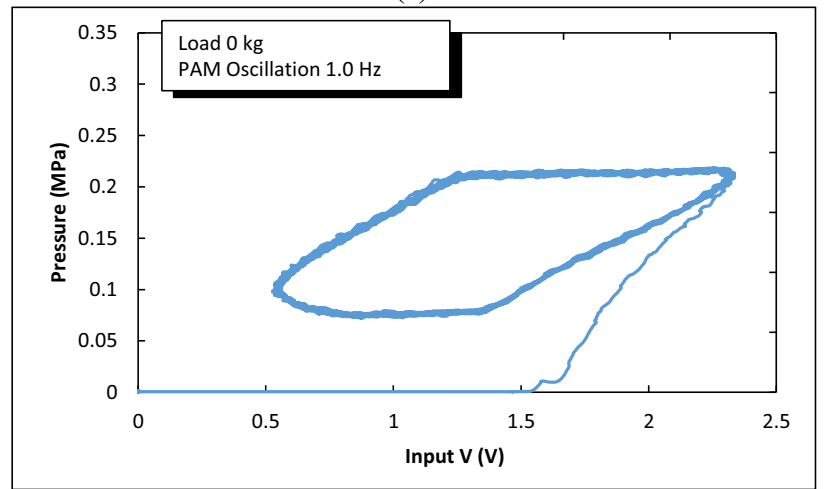

(b)

Figure 9. PAM test bed experiment with $1 \mathrm{~kg}$ and a) $0.1 \mathrm{~Hz}$ and b) $1.0 \mathrm{~Hz}$.

From the $1 \mathrm{~kg}$ load experiment in Figure 10, the displacement for $0.1 \mathrm{~Hz}$ (a) is very large, which is up to $40 \mathrm{~mm}$. At the same time, during speed increased $1.0 \mathrm{~Hz}$, the displacement is small (b), which is only at $15 \mathrm{~mm}$.
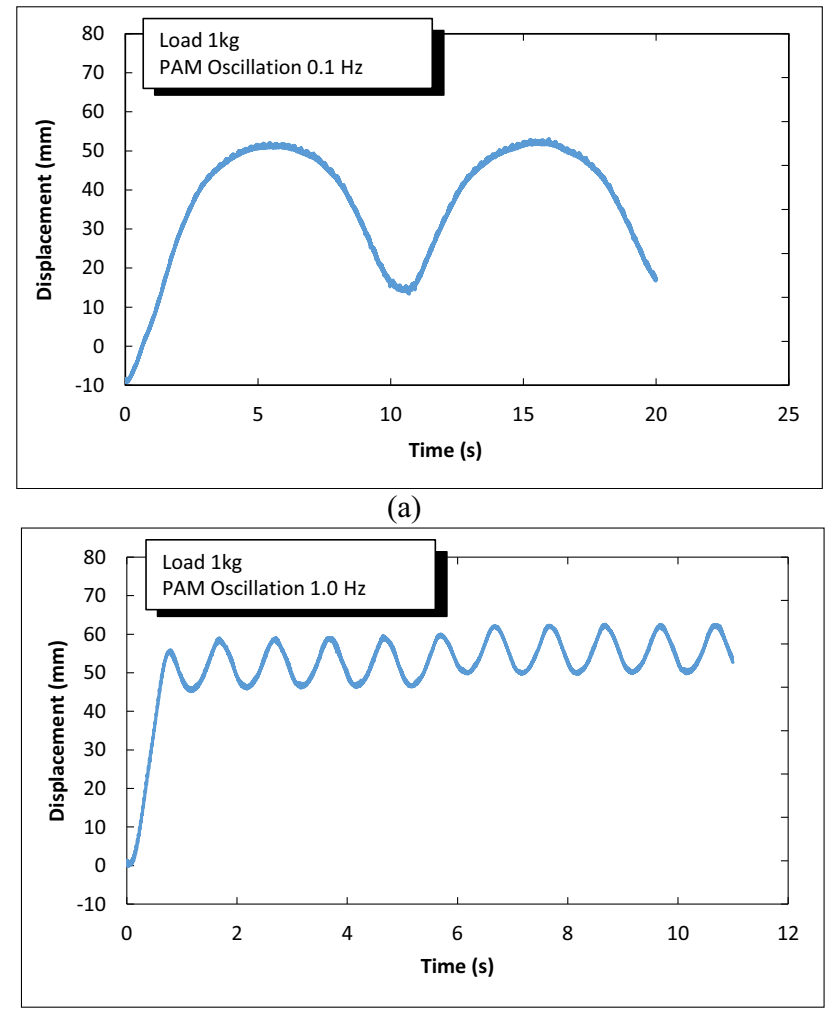

(b)

Figure 10. PAM test bed displacement experiment with $1 \mathrm{~kg}$ and a) $0.1 \mathrm{~Hz}$ and b) $1.0 \mathrm{~Hz}$.
Similar to $0 \mathrm{~kg}$ load results, $0.1 \mathrm{~Hz}$ give a linear system as shown in Figure 11(a) while the $1.0 \mathrm{~Hz}$ gives a nonlinear system in Figure 11(b) due to increase of the speed during test. Overall results show that this PAM is suitable for slow speed as the displacement is greater. Furthermore, for slow speed results, the PAM shows the linear system which is good for AFO performance.

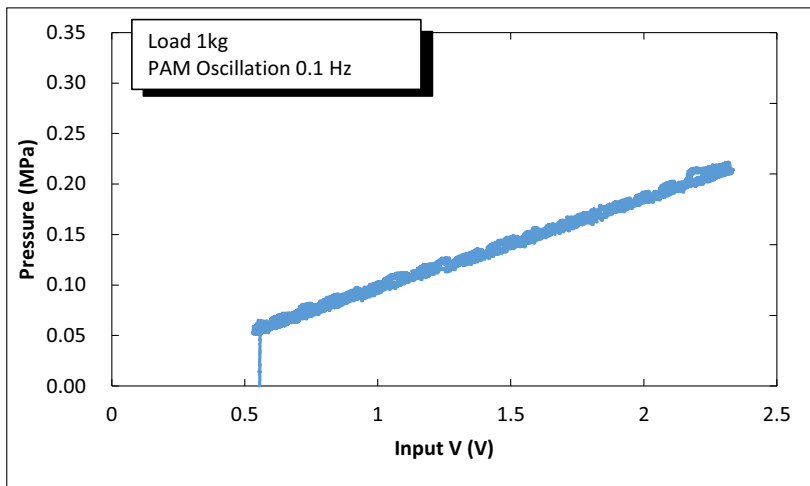

(a)

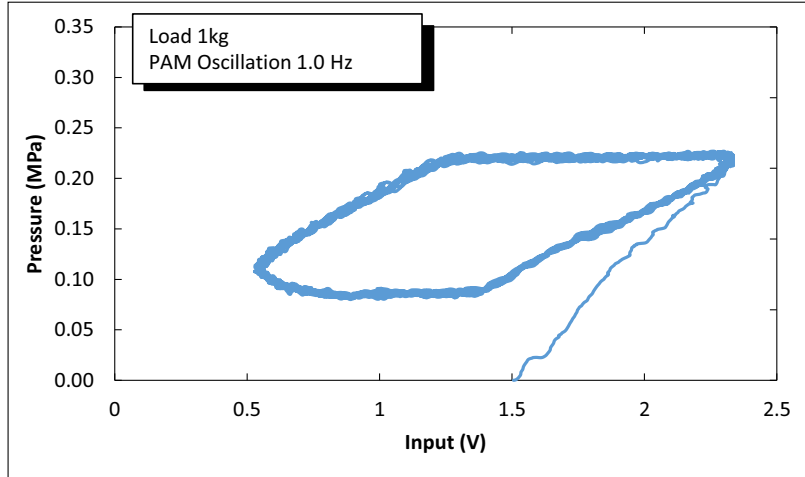

(b)

Figure 11. PAM test bed experiment with $1 \mathrm{~kg}$ and a) $0.1 \mathrm{~Hz}$ and d) $1.0 \mathrm{~Hz}$.

\section{Conclusions}

This research involved the fabrications and electronic parts assembled of a new AFO device. The needed data especially the angle moment (ankle angle) and also plantarflexion angle was recorded. From the results, the newly designed AFO actuated by PAM could be used as a potential rehabilitation intervention device for children with disabilities focusing the foot drop syndrome and inversion symptoms.

\section{Acknowledgement}

This research is funded by the Ministry of Education Malaysia under the Research Acculturation Collaboration Effort (RACE) grant [Grant no: $\mathrm{RACE} / \mathrm{c}(4) / 1333 / 2016(6)]$. The authors would like to thank Universiti Malaysia Sarawak (UNIMAS), University of Malaya (UM) and Shibaura Institute of Technology, Japan for providing the facilities for this research. 


\section{References}

1. Gottschall, J. S., \& Kram, R. 2003. Energy cost and muscular activity required for propulsion during walking. Journal of Applied Physiology, 94(5), 17661772

2. P ark, Y. L., Chen, B. R., Young, D., Stirling, L., Wood, R. J., Goldfield, E., \& Nagpal, R. 2011, September. Bio-inspired active soft orthotic device for ankle foot pathologies. In Intelligent Robots and Systems (IROS), 2011 IEEE/RSJ International Conference on (pp. 4488-4495). IEEE.

3. Shorter, K. A. 2011. The Design and Control of Active Ankle-Foot Orthoses. University of Illinois, Urbana- Champaign.

4. Abdollahi Sofla, M. 2012. Identification, simulation and control of an ankle foot orthosis (Doctoral dissertation, University of Toledo).

5. Chin, R., Hsiao-Wecksler, E. T., Loth, E., Kogler, G., Manwaring, S. D., Tyson, S. N. \& Gilmer, J. N. 2009. A pneumatic power harvesting ankle-foot orthosis to prevent foot-drop. Journal of NeuroEngineering and Rehabilitation, 6(1), 19.

6. Chen, W. P., Tang, F. T., \& Ju, C. W. 2001. Stress distribution of the foot during mid-stance to push-off in barefoot gait: a 3-D finite element analysis, Clinical Biomechanics, 16(7), 614-620.

7. Kao, P. C., \& Ferris, D. P. 2006. Motor adaptation during dorsiflexion-assisted walking with a powered orthosis. Journal of Biomechanics, 39, S31.

8. Shorter, K. A., Xia, J., Hsiao-Wecksler, E. T., Durfee, W. K., \& Kogler, G. F. 2013. Technologies for powered ankle-foot orthotic systems: Possibilities and challenges. Mechatronics, IEEE/ASME Transactions on, 18(1), 337-347.

9. Shorter, K. A. 2011. The design and control of active ankle-foot orthoses (Doctoral dissertation, University of Illinois at Urbana-Champaign).

10. Daerden, Frank, and Dirk Lefeber. "Pneumatic artificial muscles: actuators for robotics and automation." European journal of mechanical and environmental engineering 47.1 (2002): 11-21.

11. S. Mohamaddan, N.Z. Ishak, A.M.A.A.M. Mohtar, S. Yamamoto' S.Z.M. Dawal, E.B. Safawi\& H. Khamis. 2016. Design and Analysis of Ankle Foot Orthosis for Disabled Children. JurnalTeknologi. yd 